\title{
Phalangeal joints kinematics during ostrich (Struthio camelus) locomotion (\#12297)
}

Third revision

Please read the Important notes below, the Review guidance on page 2 and our Standout reviewing tips on page 3. When ready submit online. The manuscript starts on page 4.

Important notes

\section{Editor}

Virginia Abdala

Files

1 Tracked changes manuscript(s)

1 Rebuttal letter(s)

7 Figure file(s)

3 Table file(s)

1 Raw data file(s)

Please visit the overview page to download and review the files not included in this review PDF.

Involves vertebrate animals. 
Please read in full before you begin

\section{How to review}

When ready submit your review online. The review form is divided into 5 sections. Please consider these when composing your review:

\section{BASIC REPORTING}

2. EXPERIMENTAL DESIGN

3. VALIDITY OF THE FINDINGS

4. General comments

5. Confidential notes to the editor

You can also annotate this PDF and upload it as part of your review

To finish, enter your editorial recommendation (accept, revise or reject) and submit.

\section{BASIC REPORTING}

Clear, unambiguous, professional English language used throughout.

Intro \& background to show context. Literature well referenced $\&$ relevant.

Structure conforms to PeerJ standards, discipline norm, or improved for clarity.

Figures are relevant, high quality, well labelled \& described.

Raw data supplied (see PeerJ policy).

\section{EXPERIMENTAL DESIGN}

Original primary research within Scope of the journal.

Research question well defined, relevant $\&$ meaningful. It is stated how the research fills an identified knowledge gap.

Rigorous investigation performed to a high technical \& ethical standard.

Methods described with sufficient detail \& information to replicate.

\section{VALIDITY OF THE FINDINGS}

Impact and novelty not assessed. Negative/inconclusive results accepted. Meaningful replication encouraged where rationale $\&$ benefit to literature is clearly stated.

Data is robust, statistically sound, $\&$ controlled.
Conclusions are well stated, linked to original research question \& limited to supporting results.

Speculation is welcome, but should be identified as such.

The above is the editorial criteria summary. To view in full visit https://peerj.com/about/editorialcriteria/ 
The best reviewers use these techniques

Tip

\author{
Support criticisms with \\ evidence from the text or from \\ other sources
}

\section{Give specific suggestions on how to improve the manuscript}

\section{Comment on language and grammar issues}

Organize by importance of the issues, and number your points

\section{Example}

Smith et al (J of Methodology, 2005, V3, pp 123) have shown that the analysis you use in Lines 241-250 is not the most appropriate for this situation. Please explain why you used this method.

Your introduction needs more detail. I suggest that you improve the description at lines 57- 86 to provide more justification for your study (specifically, you should expand upon the knowledge gap being filled).

The English language should be improved to ensure that your international audience can clearly understand your text. I suggest that you have a native English speaking colleague review your manuscript. Some examples where the language could be improved include lines 23, 77, 121, 128 - the current phrasing makes comprehension difficult.

1. Your most important issue

2. The next most important item

3. ...

4. The least important points

Line 56: Note that experimental data on sprawling animals needs to be updated. Line 66: Please consider exchanging "modern" with "cursorial".

I thank you for providing the raw data, however your supplemental files need more descriptive metadata identifiers to be useful to future readers. Although your results are compelling, the data analysis should be improved in the following ways: $A A, B B, C C$

I commend the authors for their extensive data set, compiled over many years of detailed fieldwork. In addition, the manuscript is clearly written in professional, unambiguous language. If there is a weakness, it is in the statistical analysis (as I have noted above) which should be improved upon before Acceptance.
Comment on strengths (as well as weaknesses) of the manuscript




\title{
Phalangeal joints kinematics during ostrich (Struthio camelus) locomotion
}

\author{
Rui Zhang ${ }^{\text {Corresp., }}{ }^{1}$, Qiaoli Ji ${ }^{1}$, Gang Luo ${ }^{1}$, Shuliang Xue ${ }^{1}$, Songsong Ma ${ }^{1}$, Jianqiao Li ${ }^{1}$, Lei Ren ${ }^{\text {Corresp. }}{ }^{1,2}$ \\ ${ }^{1}$ Key Laboratory of Bionic Engineering, Ministry of Education, Jilin University, Changchun, the People's Republic of China \\ 2 School of Mechanical, Aerospace and Civil Engineering, University of Manchester, Manchester, United Kingdom \\ Corresponding Authors: Rui Zhang, Lei Ren \\ Email address: zhangrui@jlu.edu.cn, lei.ren@manchester.ac.uk
}

The ostrich is a highly cursorial bipedal land animal with a permanently elevated metatarsophalangeal joint supported by only two toes. Although locomotor kinematics in walking and running ostriches have been examined, these studies have been largely limited to above the metatarsophalangeal joint. In this study, kinematic data of all major toe joints were collected from gaits with double support (slow walking) to running during stance period in a semi-natural setup with two selected cooperative ostriches. Statistical analyses were conducted to investigate the effect of locomotor gait on toe joint kinematics. The MTP3 and MTP4 joints exhibit the largest range of motion whereas the first phalangeal joint of the 4 th toe shows the largest motion variability. The interphalangeal joints of the 3rd and 4th toes present very similar motion patterns over stance phases of slow walking and running. However, the motion patterns of the MTP3 and MTP4 joints and the vertical displacement of the metatarsophalangeal joint are significantly different during running and slow walking. Because of the biomechanical requirements, ostrich is likely to select the inverted pendulum gait at low speeds and also the bouncing gait at high speeds to improve movement performance and energy economy. Interestingly, the motions of the MTP3 and MTP4 joints are highly synchronized from slow to fast locomotion. This strongly suggests that the 3rd and 4th toes really work as an "integrated system" with the 3rd toe as the main load bearing element whilst the 4th toe as the complementary load sharing element with a primary role to ensure the lateral stability of the permanently elevated metatarsophalangeal joint. 


\section{Title page}

\section{Title: Phalangeal Joints Kinematics during Ostrich (Struthio camelus) Locomotion}

Rui Zhang ${ }^{* 1}$, Qiaoli Ji ${ }^{1}$, Gang Luo ${ }^{1}$, Shuliang Xue ${ }^{1}$, Songsong Ma ${ }^{1}$, Jianqiao $\mathrm{Li}^{1}$, Lei Ren ${ }^{* 1,2}$

${ }^{1}$ Key Laboratory of Bionic Engineering, Ministry of Education, Jilin University, Changchun, P.R. China

${ }^{2}$ School of Mechanical, Aerospace and Civil Engineering, University of Manchester, Manchester, M13 9PL, UK

${ }^{*}$ Corresponding Author:

Dr. Rui Zhang

Key Laboratory of Bionic Engineering,

Ministry of Education

Jilin University, 130022

Changchun, P.R. China

Tel. 08643185095760509

E-mail: zhangrui@jlu.edu.cn

\section{Dr. Lei Ren}

School of Mechanical, Aerospace and Civil Engineering

University of Manchester

Manchester, UK

M13 9PL

Tel. 00441613064251

Email: lei.ren@manchester.ac.uk 


\section{Abstract}

39 The ostrich is a highly cursorial bipedal land animal with a permanently elevated 40 metatarsophalangeal joint supported by only two toes. Although locomotor kinematics in walking 41 and running ostriches have been examined, these studies have been largely limited to above the metatarsophalangeal joint. In this study, kinematic data of all major toe joints were collected from gaits with double support (slow walking) to running during stance period in a semi-natural setup 44 with two selected cooperative ostriches. Statistical analyses were conducted to investigate the effect of locomotor gait on toe joint kinematics. The MTP3 and MTP4 joints exhibit the largest range of motion whereas the first phalangeal joint of the 4th toe shows the largest motion variability. The interphalangeal joints of the 3rd and 4th toes present very similar motion patterns over stance phases of slow walking and running. However, the motion patterns of the MTP3 and MTP4 joints and the vertical displacement of the metatarsophalangeal joint are significantly 50 different during running and slow walking. Because of the biomechanical requirements, ostrich is 51 likely to select the inverted pendulum gait at low speeds and also the bouncing gait at high speeds to improve movement performance and energy economy. Interestingly, the motions of the MTP3 and MTP4 joints are highly synchronized from slow to fast locomotion. This strongly suggests

54 that the 3rd and 4th toes really work as an "integrated system" with the 3rd toe as the main load bearing element whilst the 4th toe as the complementary load sharing element with a primary role to ensure the lateral stability of the permanently elevated metatarsophalangeal joint. 
Ostriches have a large number of adaptations that allow them to move both economically and quickly. They (Struthio camelus) are acknowledged as the fastest and largest extant bipedal land animal also with extraordinary endurance during locomotion and can possibly run faster than antelopes of a comparable size (Schaller et al., 2009; Alexander et al., 1979; Abourachid and Renous, 2000; Schaller et al., 2009, 2011). The ostrich has been filmed running steadily for 30 minutes at a speed exceeding $50 \mathrm{~km} / \mathrm{h}$ and moving at a speed of $70 \mathrm{~km} / \mathrm{h}$ for short sprints, with a step length reaching up to 5m (Abourachid and Renous, 2000; Schaller et al., 2011). In addition, it is also reported that they are capable of cutting maneuvers with minimal changes of their leg kinematics and joint torques (Jindrich et al., 2007). Some studies showed that ostriches are highly adapted to very economic locomotion from slow walking to fast running (Rubenson et al., 2004, 2010).

Ostrich leg morphology may provide the mechanical foundation for this unique locomotor performance (Schaller et al., 2011). For instance, compared to other large cursorial ratite birds, e.g. rhea (Rhea spp.), emu (Dromaius novaehollandiae), cassowary (Casuarius spp.), ostrich has the longest absolute limbs that contribute to achieve great step lengths and step frequency (Gatesy and Biewener, 1991). In addition, the proportion of ostrich hindlimb bones and multijointed muscle tendon system are highly adapted for locomotion. On the other hand, compared to other terrestrial birds, such as rhea (Rhea spp.), emu (Dromaius novaehollandiae) and brown kiwi (Apteryx australis), ostriches relatively erect femurs increase the joint chain extension and symmetrical movement (Abourachid and Renous, 2000). Furthermore, the ligaments system and tendons in the hindlimb joints play a vital role in ostrich gaits performance and economy. Ligamentous system of the intertarsal joint prevents tarsometatarsal rotations by providing a primary guiding function and ensuring joint coherence throughout range of motion. During stance phase, the extended intertarsal joint is sustained in the engaged state to provide additional support for body mass (Schaller et al., 2009). The distal part of their hindlimb are primarily controlled by the long and stretched tendons; therefore, the metatarsophalangeal joint may play an important role in storing and releasing elastic energy, and absorbing shock during fast locomotion hence providing an energy-saving mechanism (Alexander 1984, 1985; Gatesy, 1991; Castanet et al., 2000; Almeida Paz et al., 2008). 
87 It is noteworthy that unique adaptations are also evident in ostrich toe morphology. While 88 ordinary birds have three or four toes, the ostrich has only two toes, the main 3rd toe and the lateral 4th toe. Another unique adaptation at the distal part of the hindlimb is the supra-jointed toe posture with the metatarsophalangeal joint and proximal phalanx of both toes being permanently elevated above the ground surface (Schaller, et al., 2011; Deeming, 2003). Pressure plate data suggested that both toes play a vital role in ostrich terrestrial locomotion with different load distributions in walking and running. The 3rd toe sustains most of the ground reaction force during locomotion and its claw provides the forces at push-off in fast locomotion. In addition, the 4th toe functions as a lateral support during locomotion (Schaller et al., 2007, 2011; Schaller, N. U. Structural attributes contributing to locomotor performance in the ostrich (Struthio camelus), $\mathrm{PhD}$ Thesis, University of Heidelberg, Heidelberg, Germany, 2008.). The only rigid element in ostrich toes is the single remaining claw that functions as a positional anchor at fast speed when embedded in the terrain (Schaller et al., 2011). The major tendons are distributed in tibiotarsus, tarsometatarsus and two digits (Gangl et al,. 2004). There is an interphalangeal ligament inserting mediodistally at the proximal phalanx of the 3rd toe and medioproximally at the second phalanx of the 4th toe (Schaller et al., 2011). This ligament couples the toes motion and limits the 4th toe abduction to the 3rd toe main direction. Therefore, ostrich toes may execute locomotion through the movement coordination with each other and form an "integrated system".

Although a large number of studies have been conducted to investigate the ostrich hindlimb kinematics during locomotion (Haughton, 1865; Alexander et al., 1979; Alexander, 1985; Gatesy and Biewener, 1991; Abourachid and Renous, 2000; Jindrich et al., 2007; Rubenson et al., 2004, 2007, 2010; Watson et al., 2011; Smith et al., 2006, 2007, 2010, 2013; Schaller et al., 2009, 2011; Birn-Jeffery et al., 2014; Hutchinson et al., 2015, 2016), those kinematic analysis are mainly focused on hip, knee, the metatarsophalangeal and intertarsal joints. So far, little is known about the relative motions of the $3 \mathrm{rd}$ and 4 th toes intrinsic joints during ostrich foot locomotion.

112 As the only body part in contact with the ground surface, the unique toe joint motions may play 113 important biomechanical roles during locomotion. Therefore, a better understanding of the 114 interphalangeal joint of toes and the metatarsophalangeal joint kinematics may provide valuable 115 information to reveal the biomechanical mechanism underlying the extraordinary locomotor 116 performance of ostriches. Our primary aims were to test the hypothesis that the 3rd and 4th toes 
117 work as an "integrated system", and motions of the metatarsophalangeal joint and the

118 interphalangeal joints of the 3rd and 4th toes have significantly different patterns during slow

119 walking and running gaits.

120 In this study, we examined the kinematics of all major joints of ostrich toes in vivo during slow

121 walking and running using high speed videos and specially designed markers. This included the

122 interphalangeal joint motions within both toes, the relative motions between the first phalanx of

123 the 3rd and 4th toe with respect to the tarsometatarsus, and the angle between the long axis of the

$1243 \mathrm{rd}$ and 4 th toe over entire stance phases. Statistical analysis was also conducted to investigate

125 the effect of locomotor gait on those joint motions. This study aimed to investigate whether there

126 were differences between the two toes joint motions in slow walking and running. To test our

127 hypothesis that phalangeal joint angle and the vertical displacement of the metatarsophalangeal

128 joint at touch-down, mid-stance, lift-off, joint range of motion, maximum and minimum joint

129 angles were selected as key indicators for statistical tests.

130 Materials and Methods

131 Animals

132 Ten healthy sub-adult ostriches (Struthio camelus) with an average age of eight months were 133 selected from the Ji'an breeder, Jilin province, P.R. China. The average mass and height of these 134 ostriches are $84.5 \pm 2.12 \mathrm{~kg}$ and $2.11 \pm 0.01 \mathrm{~m}$ (displayed by means \pm S.D), respectively. Without any 135 form of surgical treatment or invasive physical manipulation, the individuals were in excellent 136 physical condition with the properly elevated metatarsophalangeal joints, which represented the 137 average body proportion and weight for ostriches of their age and sex (Deeming, 2003). These 138 ostriches were kept in outdoor enclosure in daytime with unlimited access to food and water, and 139 housed in an indoor enclosure at night. Each bird was trained to walk and run on a fenced-in 140 corridor at least 30 minutes each time, twice per day over a month before data collection. After 141 comprehensive comparison of representation and amenability, two tractable female sub-adult 142 ostriches were selected as objects to complete all tests. All living and experimental conditions 143 were approved by the Institutional Animal Care and Use Committee (IACUC, protocol number: 144 20140706) of Jilin University, P.R. China.

\section{Experimental setup and trials}


146 A $80 \mathrm{~m}$ long runway fenced by $1.5 \mathrm{~m}$ tall wire mesh was set up in the breeding field with a data 147 acquisition area in the middle of $4 \mathrm{~m}$ long and $1 \mathrm{~m}$ wide zone (see Figure 1). The runway was 148 covered with a $3 \mathrm{~mm}$ non-slip rubber sheet to prevent potential damage from ostrich foot. At both 149 ends of the runway, large spaces were provided for the ostriches to rest and eat. The area outside 150 of the data acquisition zone was about $76 \mathrm{~m}$ long and $2 \mathrm{~m}$ wide with two "V" shape transition areas 151 gradually connecting to the data acquisition zone, which helps guide the ostriches to naturally 152 enter into the data acquisition area. A high-speed video system with three synchronized digital 153 cameras (Casio Exilim EX-FH25, Tokyo, Japan; 240 frames $\mathrm{s}^{-1}$ ) was placed around the central 154 zone of the data acquisition area in a triangle shape with one camera positioned perpendicular to 155 the sagittal plane of motion (see Figure 1).

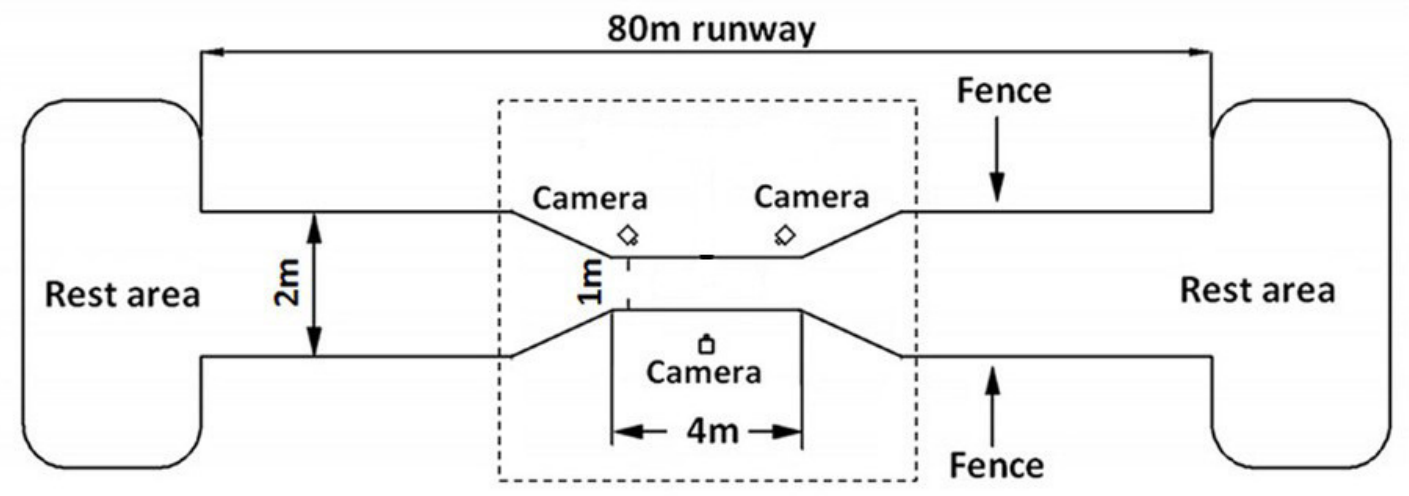

156 Figure 1. Schematic diagram of the experimental site of $80 \mathrm{~m}$ long. The data acquisition area in the center of the dotted box is of $4 \mathrm{~m}$ long and $1 \mathrm{~m}$ wide. Fences of $1.5 \mathrm{~m}$ high were set on both sides of the runway. Three high-speed cameras were placed in the central data acquisition area in a triangular shape. Both ends of runway are rest areas for ostriches to rest and eat foods.

During measurements, ostriches were led by their breeders or experimenters, using positive reinforcement such as food rewards and vocal commands, with the goal of maintaining a steady speed across a straight distance of about $15 \mathrm{~m}$. Experimenters randomly varied the speed from slow walking to fast running across trials and allowed ample rest and food between trials to prevent fatigue. Experiments were cancelled if animals showed fatigue that would cause discomfort or adversely affect our measurements. To minimize the interference of sunlight, one sunshade net was set on the top of the data acquisition zone.

\section{Marker placements and joint angles}


168 Nine specially designed thermoplastic plates carrying nine retro-reflective markers were firmly mounted at the major anatomical landmarks around the ostrich left foot toes using double sided tapes (see Figure 2A). The marker locations were determined by palpation and referring to a three-dimensional (3D) geometric model of the tarsometatarsus bone and the phalanges of the $3 \mathrm{rd}$ and 4th toes, reconstructed from the CT images of a healthy adult female ostrich (Age: 3 years, Weight: 95kg, Height: 2.10m) left foot by using Mimics 10.0 software (Materialise, Leuven, Belgium) (see Figure 2B). Four markers were used for the 3rd toe at the dorsal base of the toenail (marker A), the joint between phalanges II and III (marker B), the joint between phalanges I and II (marker C), and the joint between phalanx I and tarsometatarsus (marker D). Whereas, three markers were placed on the 4th toe at the joint between phalanx I and tarsometatarsus (marker F), the joint between phalanges I and II (marker G), and the distal end of the 4th toe (marker H). Additionally, one marker was put on the anterior side of the tarsometatarsus bone proximal to the metatarsophalangeal joint (marker E). Here, the toenail, phalanges III and IV of the 3rd toe were considered as one segment (phalanges III in Figure 2B), and the phalanges II III IV and V of the 4th toe were assumed as one part (phalanges II in Figure 2B) because these phalanges are small and the relative motions among them are hard to measure and observe (Fowler et al., 1991).
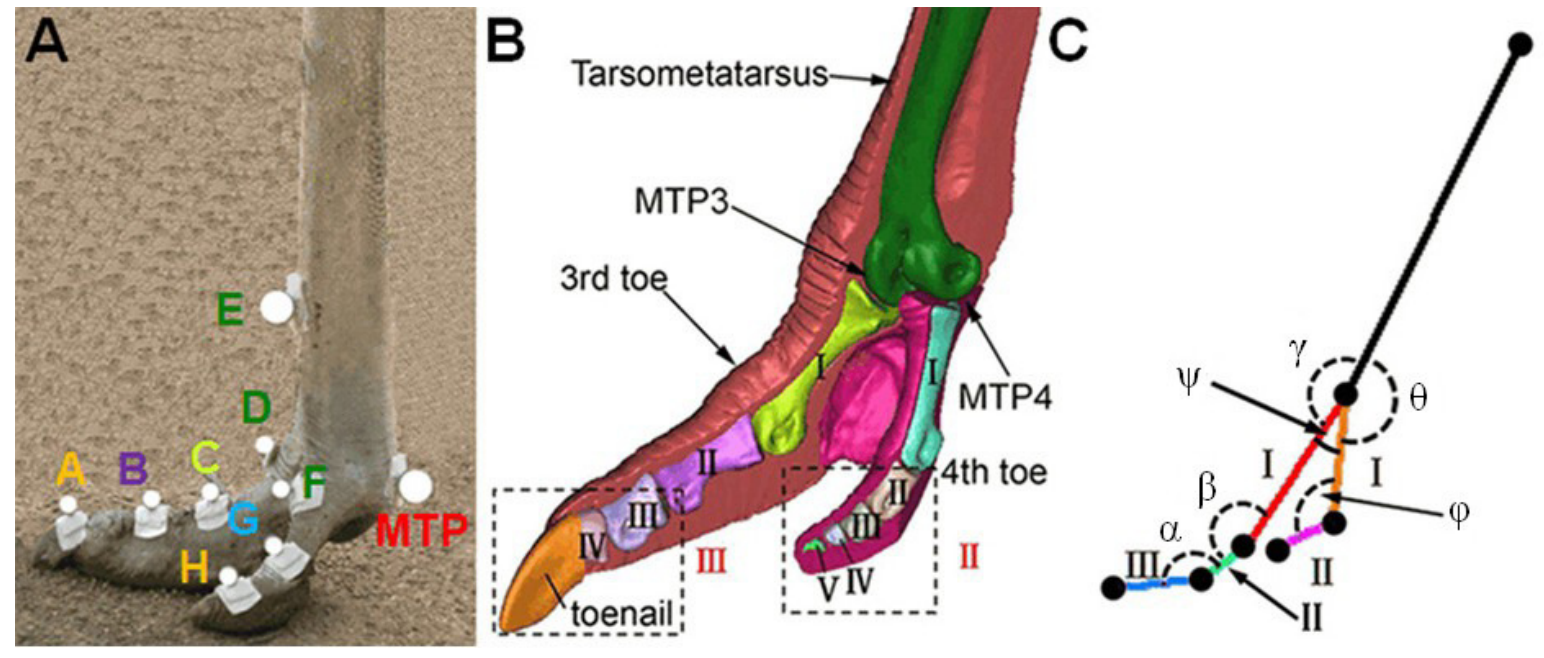

Figure 2. The reflective markers on ostrich foot and the toe joint angle measured. Nine reflective markers were placed at the major anatomical landmarks of ostrich toes (Figure 2A). The marker locations were determined by palpation and referring to a 3D geometric toe model reconstructed from the $\mathrm{CT}$ images of a healthy adult female ostrich (Age: 3 years, Weight: $95 \mathrm{~kg}$, Height: $2.10 \mathrm{~m}$ ) left foot (Figure 2B). Six toe joint angles were defined (Figure 2C): angle $\alpha$ between the phalanges II and IIIof the 3rd toe, angle $\beta$ between the phalanges I and II of the 3rd toe, angle $\gamma$ between the tarsometatarsus and the phalanx I of the 3rd toe (MTP3 joint), angle $\theta$ between the tarsometatarsus and the phalanx I of the 4th toe (MTP4 joint), angle $\varphi$ between the phalanges I and II of the 4th toe, angle $\psi$ was between the first phalanges of the 3rd and 4th toes and all angles 
192 were spatial 3D angles. MTP3 represents the joint between tarsometatarsus and the phalanx I of the 3rd toe.

193 MTP4 represents the joint between the tarsometatarsus and the phalanx I of the 4th toe. MTP represents the 194 metatarsophalangeal joint. MTP joint include the MTP3 and MTP4 joints.

195 The 3D coordinates of the nine retro-reflective markers were measured at $240 \mathrm{~Hz}$ using a three196 camera (Casio Exilim EX-FH25, Tokyo, Japan) motion tracking system (Simi Motion 2D/3D ${ }^{\circledR}$ 1977.5 software, SIMI Reality Motion Systems GmbH, Germany). Two series of representative video 198 frames recorded for slow walking and running respectively are shown in Figure 3 . The marker 199 data and joint kinematics were analyzed using Simi Motion 2D/3D ${ }^{\circledR} 7.5$ software. The software 200 allows for three-dimensional calibration, digitization of bony landmarks and calculation of the 201 segment and joint kinematic parameters of interest (Stoessel and Fischer, 2012). The average 202 error of motion tracking system measurement accuracy was $+/-1.0 \mathrm{~mm}$. The time histories of six joint angles were calculated, namely, angle $\alpha$ between the phalanges II and III of the 3rd toe,

204 angle $\beta$ between the phalanges I and II of the 3rd toe, angle $\gamma$ between the tarsometatarsus and the 205 phalanx I of the 3rd toe (MTP3 joint), angle $\theta$ between the tarsometatarsus and the phalanx I of 206 the 4th toe (MTP4 joint), angle $\varphi$ between the phalanges I and II of the 4th toe, and angle $\psi$ 207 between the first phalanges of the $3 \mathrm{rd}$ and 4 th toes (see Figure 2C). Also, we measured 208 displacements of the metatarsophalangeal joint $z$ and directly exported data by motion tracking 209 system.
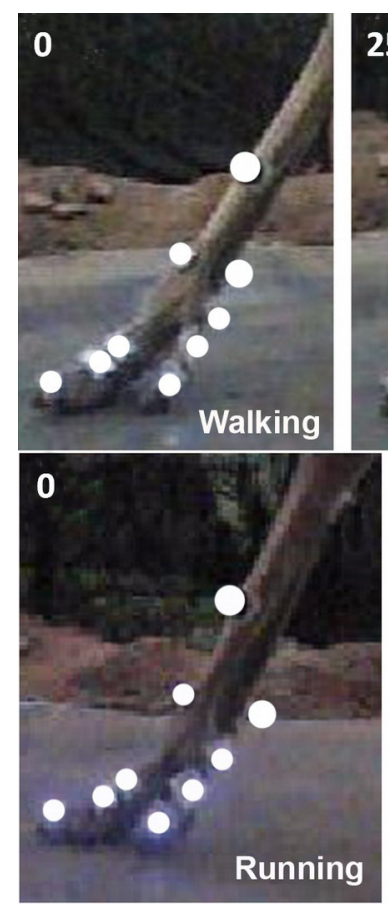
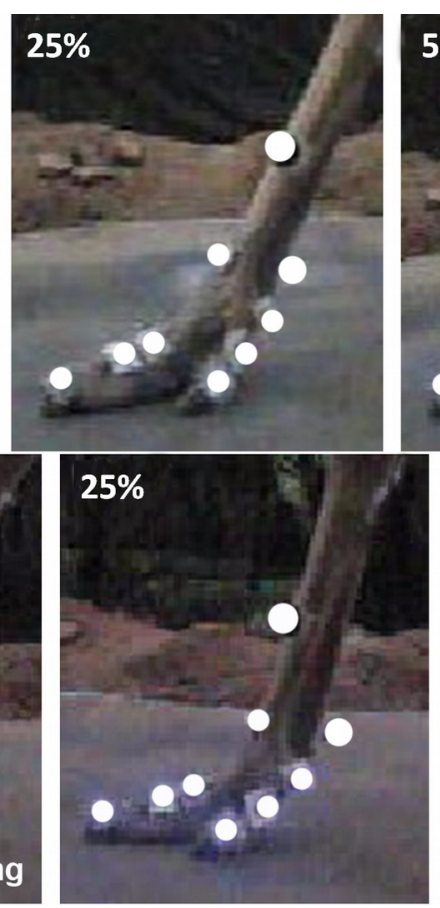
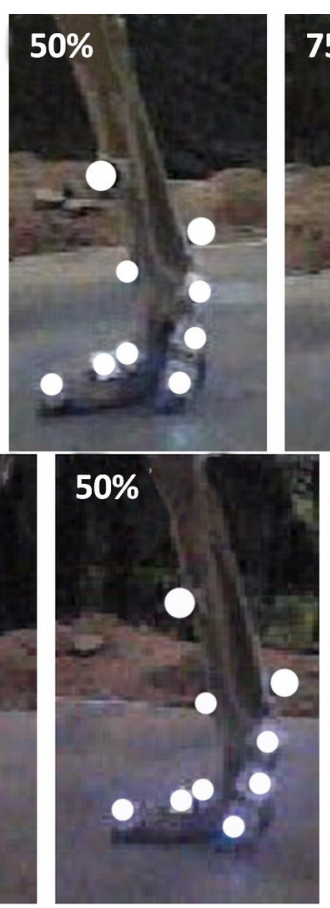
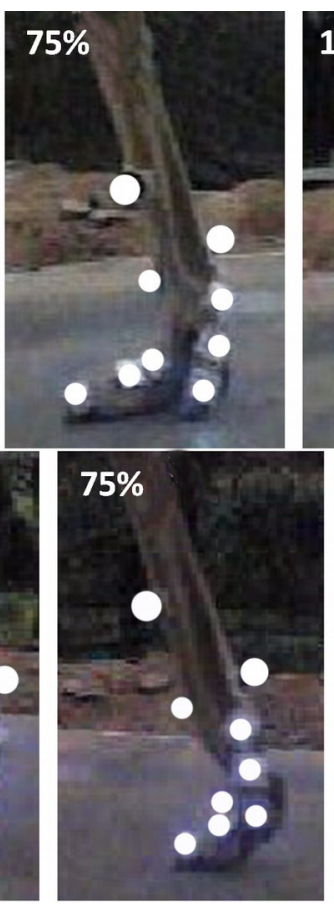
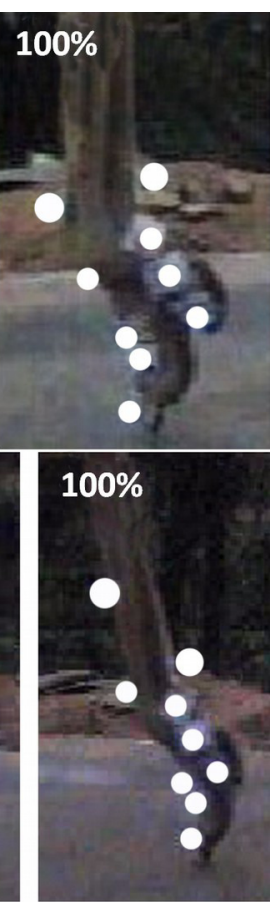

210 Figure 3. Two representative high speed video traces of toe motions during slow walking and running in 
211 stance phases. The traces started at touchdowns when the $3 \mathrm{rd}$ toe touched the ground at $0 \%$ of stance phase. In 212 the slow walking and running trials, the mid-stance is at $50 \%$ of stance phase and the $3 \mathrm{rd}$ toe cleared off the 213 ground at $100 \%$ of stance phase.

214 Animal forward average velocity was calculated by stride length divided by stride period. We 215 defined steady state trials as those in which the absolute difference between the forward velocities 216 at two consecutive touch downs was less than $20 \%$ of the average forward velocity. Trials with 217 greater or smaller values of acceleration/deceleration were discarded. Froude numbers $\mathrm{Fr}_{r}=v^{2} /(g h)$ 218 and dimensionless speed $\left(u=F r^{0.5}\right)$ were calculated to normalize speeds, where $v$ is the forward 219 velocity of the animal, $g$ is the gravitational-acceleration constant and $h$ is the length of the 220 pendulum (leg length from hip to ground) (e.g. Alexander and Jayes, 1983). Gait parameters, 221 including, cycle period, stance duration, swing duration, duty factor and stride length, were 222 calculated for each steady state trial.

\section{Statistical analysis}

224 Statistical analyses were conducted to examine the differences in four gait parameters (stance and swing duration, cycle period and stride length), six key indicators (phalangeal joint angle/the vertical displacement of the metatarsophalangeal joint at touch-down, mid-stance, lift-off, maximum, minimum and range of motion) between slow walking and running gaits using Origin Pro 2015 software (OriginLab Corporation, Northampton, USA). In this study, trials with stance duration $>0.9 \mathrm{~s}$ and duty factor $>0.5$ were considered as walking gaits at slower speeds with double support, whereas trials with stance duration $<0.9 \mathrm{~s}$ and duty factor $<0.5$ were regarded as running gaits (Schaller et al., 2011). We used one-way ANOVA statistical technique to analyze the effect of locomotor gait on each gait parameter or joint angle/displacement indicator (Schache, 2011; Stoessel and Fischer, 2012). Using the F-test to test whether these two variations are significantly different. Statistical significance level was considered as $\mathrm{P}<0.05$.

235

236

237

238

239

240

An equal number of stance phases were included in the statistical analysis from each individual for both slow walking and running in order to weight each evenly. Additionally, in order to study the potential for inter-subject variation, interphalangeal joint angle values of slow walking and running trials from each individual were conducted to an analysis of variance. A total of 38 samples (individual A, 19 samples; individual B, 19 samples), divided between slow walking and running trials, were included in the statistical analysis (see Table 1). 

running gaits

\begin{tabular}{ccccc}
\hline & \multicolumn{2}{c}{ Slow walking trials } & \multicolumn{2}{c}{ Running trials } \\
\hline Individuals & $\mathrm{A}$ & $\mathrm{B}$ & $\mathrm{A}$ & $\mathrm{B}$ \\
Valid stance phase & 15 & 12 & 7 & 9 \\
Statistical analysis trials & 12 & 12 & 7 & 7 \\
\hline
\end{tabular}

\section{Results}

\section{Gait parameters}

245 Averages and standard derivations of key gait parameters, including stance duration, swing 246 duration, cycle period and stride length of all slow walking and running gaits were listed in Table 2472 separately. It can be seen that there were statistically significant differences in stance duration, 248 cycle period and stride length between slow walking and running gaits. Ostriches use considerably shorter cycle periods and stance duration during running than those during slow

250 walking, whereas dramatically increase their stride lengths (Abourachid and Renous, 2000). 251 There was no statistically significant difference was found in swing duration between slow 252 walking and running gaits. These observation were consistent with previous observation 253 (Alexander et al., 1979; Rubenson et al., 2004).

Table 2. The key gait parameters during slow walking and running gaits

\begin{tabular}{lcc}
\hline \multicolumn{1}{c}{ Gait parameters } & $\begin{array}{c}\text { slow walking } \\
(0.38-1.23 \mathrm{~m} / \mathrm{s})\end{array}$ & $\begin{array}{c}\text { running } \\
(2.26-3.31 \mathrm{~m} / \mathrm{s})\end{array}$ \\
\hline Number of trials & 56 & 25 \\
Statistical analysis stance phases & 24 & 14 \\
Average speed (m/s) & $0.84 \pm 0.20^{*}$ & $2.77 \pm 0.28^{*}$ \\
Froude numbers & $0.06 \pm 0.03^{*}$ & $0.66 \pm 0.13^{*}$ \\
Duty factor & $0.74 \pm 0.09^{*}$ & $0.45 \pm 0.03^{*}$ \\
Stance phase (second) & $1.22 \pm 0.33^{*}$ & $0.34 \pm 0.03^{*}$ \\
Swing phase (second) & $0.44 \pm 0.16$ & $0.42 \pm 0.02$ \\
Cycle period (second) & $1.66 \pm 0.30^{*}$ & $0.76 \pm 0.03^{*}$ \\
Stride length (meter) & $1.33 \pm 0.16^{*}$ & $2.11 \pm 0.15^{*}$ \\
\hline es are means \pm S.D. Statistically significant speed effects are indicated by an asterisk $(\mathrm{P}<$
\end{tabular}




\section{Toe joint kinematics}

258 Figure 4 showed the averages and one standard deviation zones of the six toe joint angles and the

259 vertical displacements of metatarsophalangeal joint $(\alpha, \beta, \gamma, \theta, \varphi, \psi, z)$ over the stance phases for 260 all slow walking and running trials respectively. From Figure 4A, it can be seen that the time 261 trajectories of the third phalangeal joint angle of the 3 rd toe $(\alpha)$ shared very similar patterns in 262 the stance phases during slow walking and running. The third phalangeal joint of the 3rd toe 263 extended about 10 degree immediately after the touch-down, and thereafter remained at about 264165 degree throughout from early stance to late stance. This was followed by a swift flexion of 265 about 35 degree and also a rapid extension of about 20 degree just before lift-off. However, 266 compared to slow walking gaits, it appeared that during running the joint extension in the early 267 stance finished slightly later (at $20 \%$ of the stance phase), and the joint flexion and protraction in 268 the late stance occurred earlier (at $70 \%$ of the stance phase). 

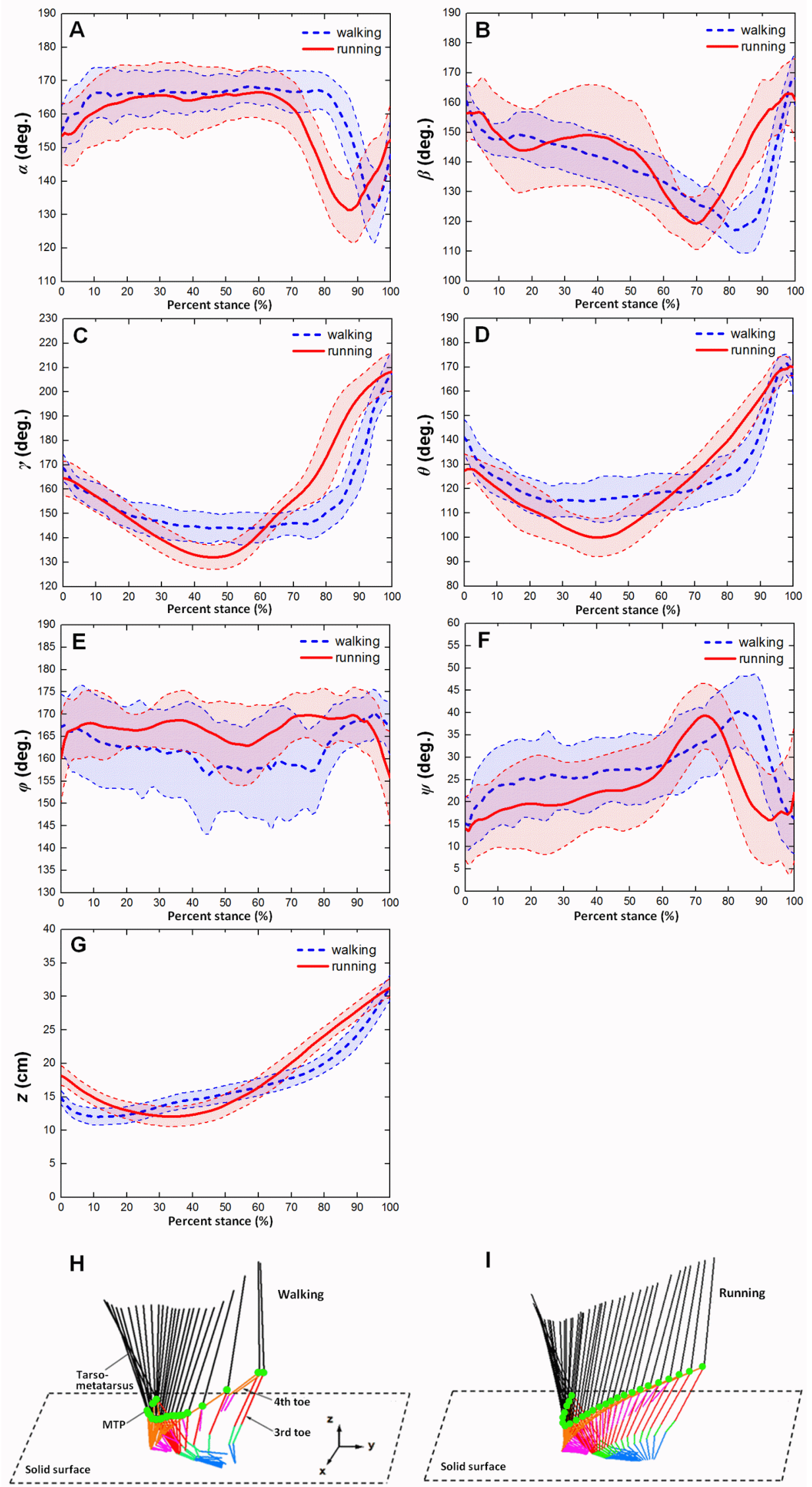
displacement of the metatarsophalangeal joint $(\alpha, \beta, \gamma, \theta, \varphi, \psi, z)$ (corresponding to A B C D E F G, respectively)over the stance phases for all slow walking (blue dotted line) and running trials (red solid line). Angle decrease represents flexion while angle increase indicates extension. Stick figure at the bottom of Figure 4H and 4I showed the ostrich foot motion in stance phase and the green point indicates the metatarsophalangeal joint.

275 From Figure 4B, we could see that the second phalangeal joint angle of the 3rd toe ( $\beta$ ) showed similar patterns in the stance phases of slow walking and running. The joint angle decreased after touch-down from about 160 degree to 120 degree or so at late stance. Thereafter, the joint extended swiftly back to about 160 degree just before lift-off. However, it stoteworthy that the joint flexed and extended much more radically in running than during slow walking, and high variability was observed from early stance to middle stance (from touch-down to $60 \%$ of stance phase).

The MTP3 joint angle $\gamma$ was been shown in Figure 4C present noticeably different patterns during slow walking from running. In slow walking, the joint angle decreased gradually from touchdown to $30 \%$ of stance phase about 25 degree, and then remained steady till reaching $80 \%$ of stance phase. A sharp joint angle increased occurs just before lift-off with the MTP3 joint extended almost 60 degree. Whereas, in running there was no plateau stage in middle stance. The joint flexed gradually from touch-down to middle stance, and thereafter extended progressively to 210 degree at lift-off.

The MTP4 joint angle $\theta$ also showed different motion patterns during slow walking from running (see Figure 4D). In slow walking, the MTP4 joint flexed about 25 degree directly after touchdown, and remained reasonably steady from early stance through to late stance. Just before liftoff, a swift joint extension occurred at the MTP4 joint reaching a nearly fully extended position at 170 degree. Whereas, in running gaits, there was no steady stage in the middle of stance phase. After touch-down, the MTP4 joint flexed gradually about 30 degree till middle stance, and thereafter followed by a progressive joint extension of 60 degree till lift-off.

The largest angle variability among all the six toe joints was observed at the first phalangeal joint angle of the 4th toe $(\varphi)$. From Figure 4E, we can see that no apparent patterns presented for angle $\varphi$ during both slow walking and running. The joint angle fluctuated around 165 degree though it appeared that larger variability occurred during slow walking rather than running. 
300 While, angle $(\psi)$ between the first phalanges of 3rd and 4th toes showed clear patterns over the 301 stance phase (see Figure 4F). The angle between the two toes moved similarly during slow 302 walking and running with a gradually increasing the 4th toe abduction to the 3rd toe main axis 303 from touch-down to late stance followed by a swift adduction before lift-off. The average peak 304 joint extension was about 39 degree for both slow walking and running. This is nearly consistent 305 with previous study that the maximum motion range angle between the 3rd and 4th toe main axes 306 was 34 degree (Schaller et al., 2011).

307 Figure 4G showed the average and one standard deviation zone of the vertical displacements of 308 the metatarsophalangeal joint $(z)$ over stance phases for all slow walking trials and for all running 309 trials respectively. It can be seen that markedly different patterns were present during slow walking compared to running. In slow walking, the metatarsophalangeal joint moved downwards towards the ground surface about $3.0 \mathrm{~cm}$ just after touch-down, and thereafter went smoothly

312 upwards about $20 \mathrm{~cm}$ before lift-off. Whereas, during running, the joint only moved downwards 313 slightly about $6.2 \mathrm{~cm}$ from touch-down to near middle stance, and then kept going upwards 314 before lift-off about $20 \mathrm{~cm}$. This was nearly consistent with Figure $4 \mathrm{H}$ and $4 \mathrm{I}$ that displayed 315 motion trajectory of ostrich foot and the metatarsophalangeal joint during slow walking and 316 running gaits. We can see that the motion trajectory during running gait was more smooth than 317 that in slow walking gait. 

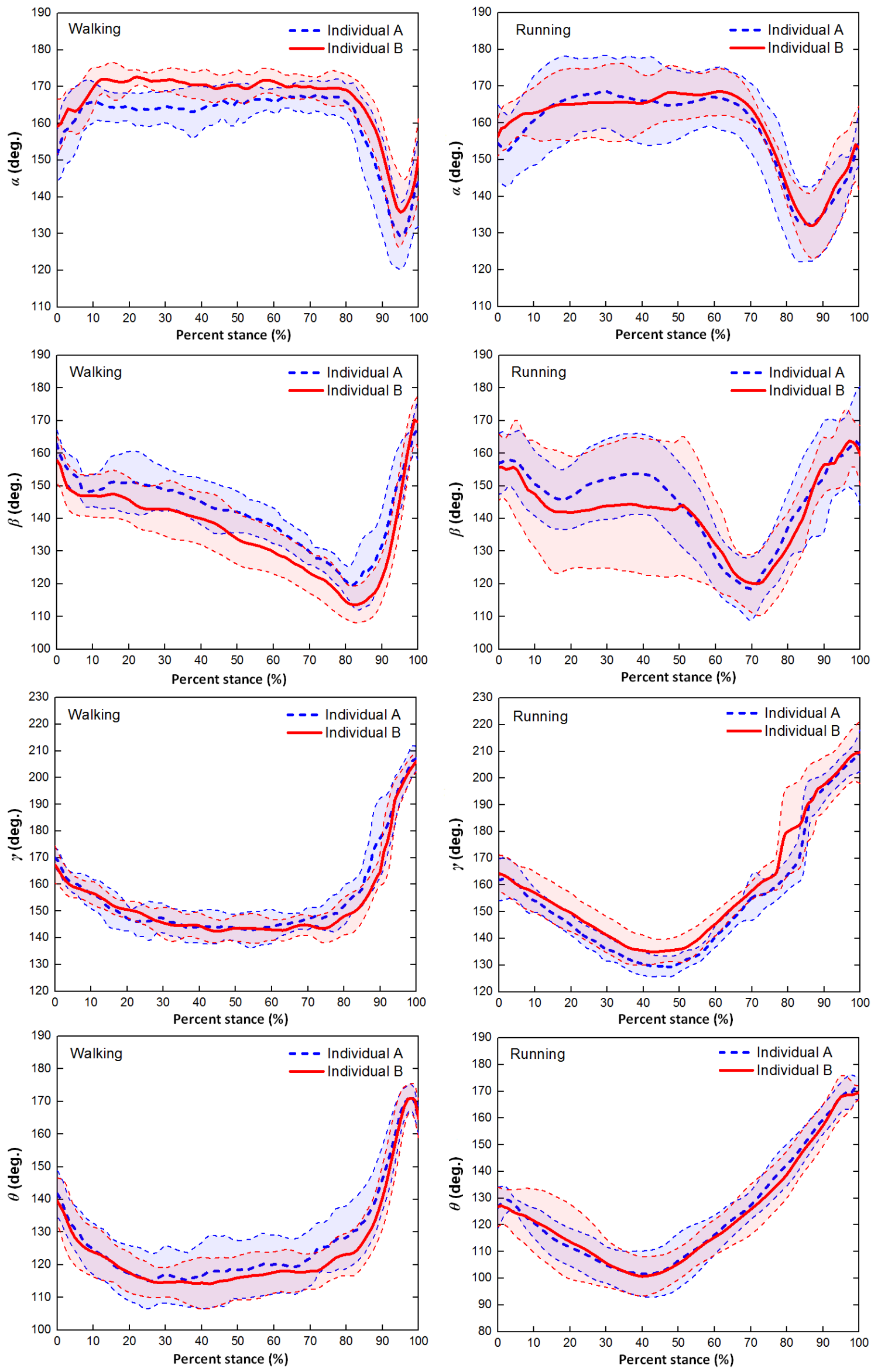

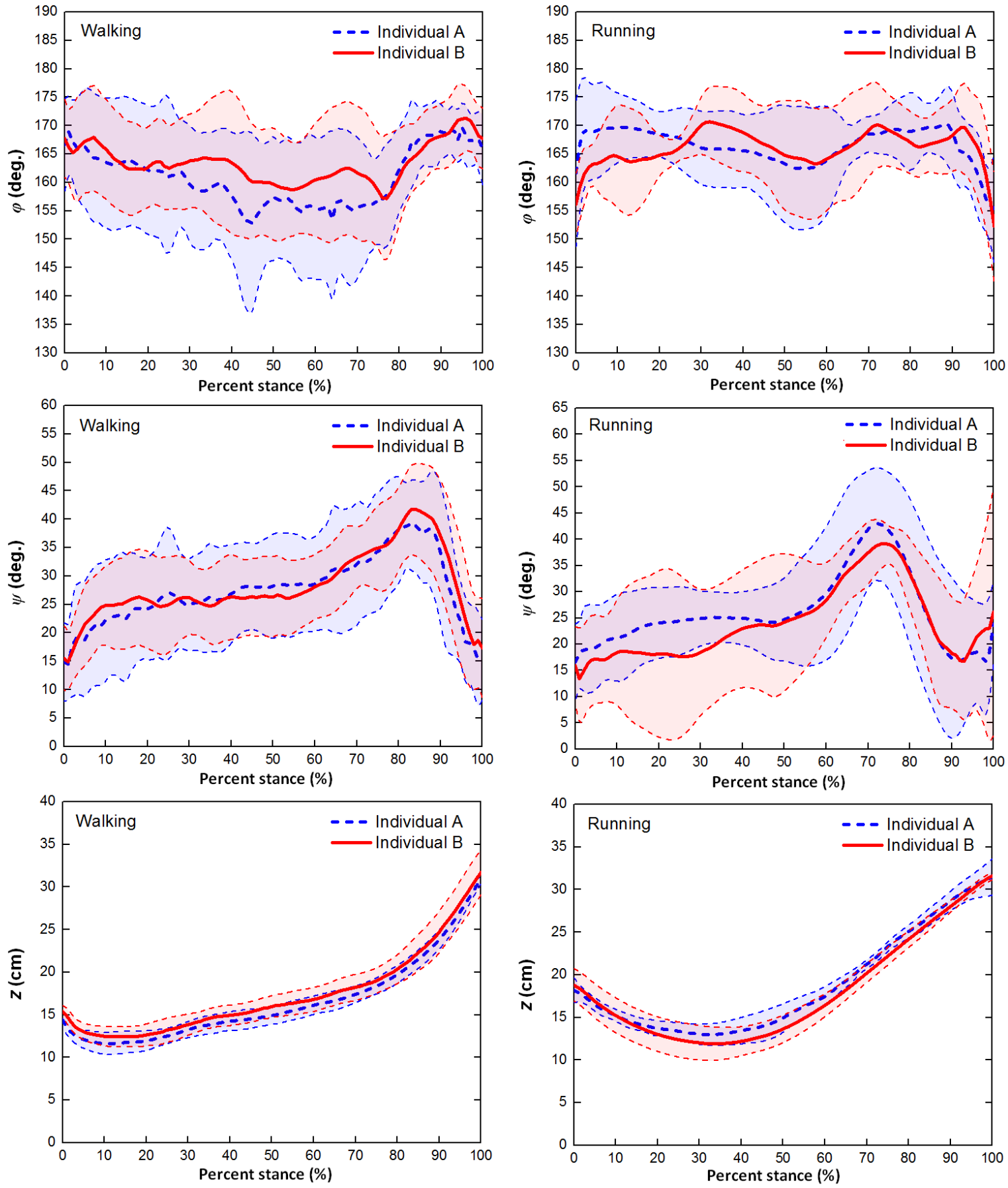

318 Figure 5. The averages and one standard deviation zones of the six toe joint angles and the vertical displacement of the metatarsophalangeal joint $(\alpha, \beta, \gamma, \theta, \varphi, \psi, z)$ over the stance phases for all slow walking and running trials. Individual A were shown in blue dotted line and individual B were shown in red solid line. 
322 Figure 5 showed that the six toe joint angles and the vertical displacement of the 323 metatarsophalangeal joint had similar motion patterns over stance phase during slow walking and 324 running gaits between individual A and individual B. 

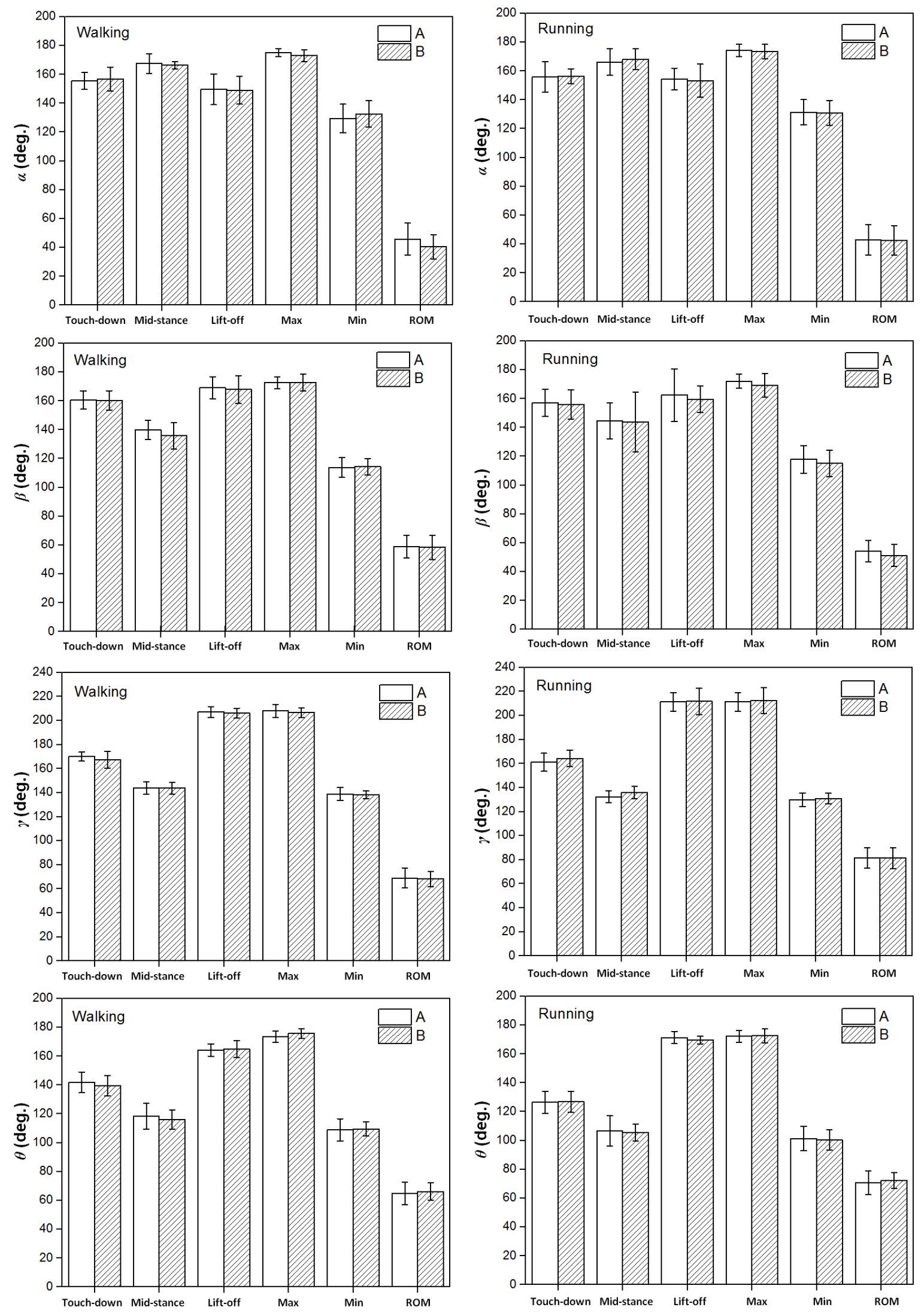

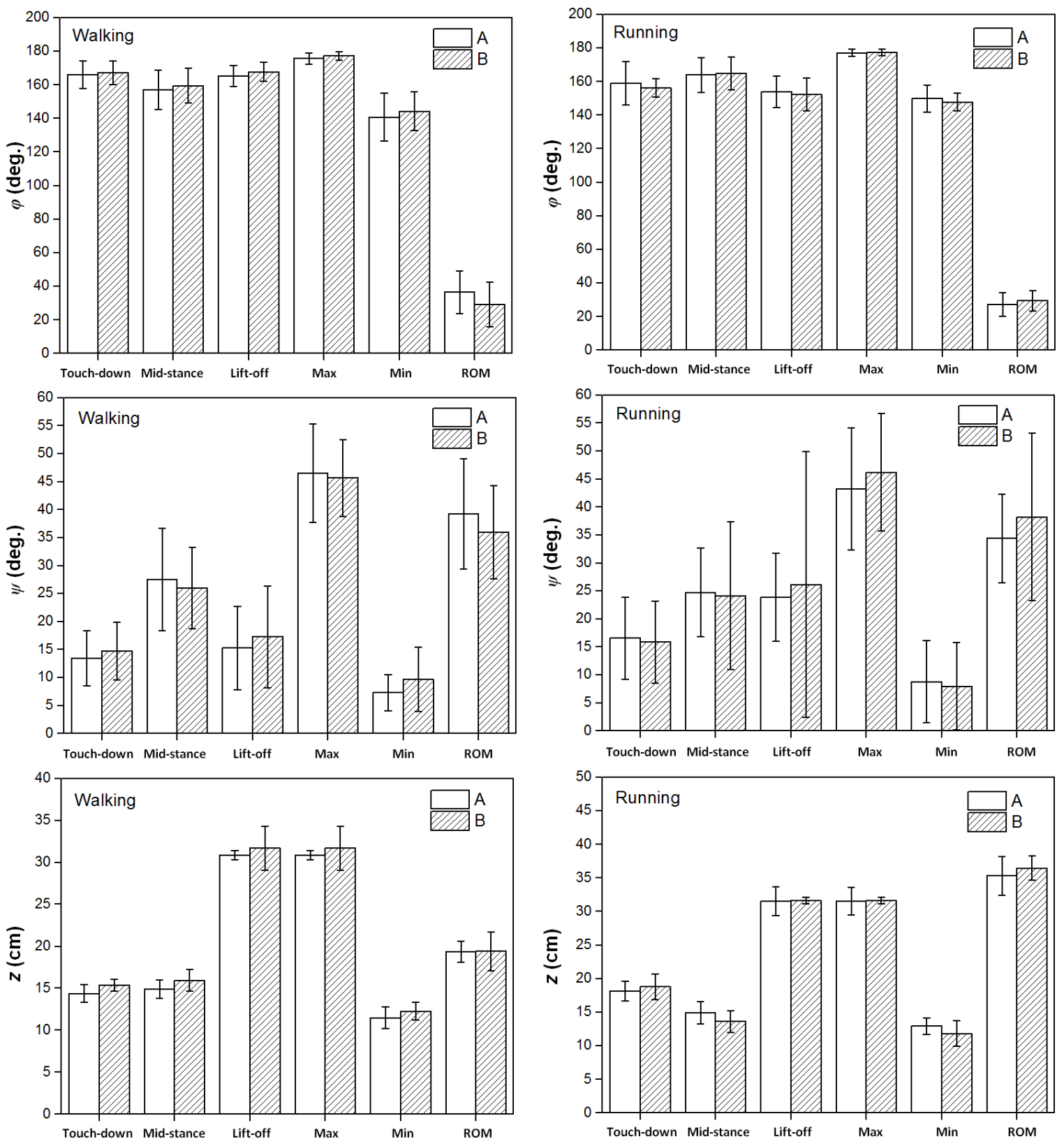

Figure 6. The averages and standard deviations of the six toe joint angles and the vertical displacement of the metatarsophalangeal joint at touch-down, mid-stance, lift-off and also the ranges of motion during slow walking and running between individual $A$ and individual $B$. Statistically significant effect of individual difference are indicated by an asterisk $(\mathrm{P}<0.05)$. No significant inter-individual effects were found, since there are no * in the figure.

330 Figure 6 showed that no statistically significant inter-individual differences were found in the six

331 toe joint angles and the vertical displacement of the metatarsophalangeal joint at touch-down, 332 mid-stance, lift-off and also the ranges of motion during slow walking and running gaits. 


\section{Effect of locomotor gait}

334 The results of the statistical analysis examining the effect of locomotor gait on the six key 335 indicators (angles/displacements at touch-down, mid-stance, lift-off, maximum, minimum and 336 range of motion) of the six toe joint angles and the vertical displacement of the 337 metatarsophalangeal joint were listed in Figure 7. Among all the six toe joints, the MTP3 and 338 MTP4 joints showed the largest ranges of motion. Whereas, angle $\varphi$ had the smallest range of 339 motion. As shown in Figure 7, no statistically significant differences were found for the six key

340 indicators of angles $\alpha, \beta, \varphi, \psi$ between slow walking and running gaits. Statistically significant 341 differences were found for the range of motion of the second phalangeal joint angle of the 3 rd toe $342(\beta)$ and the lift-off angle of the first phalangeal joint angle of the 4th toe $(\varphi)$. A slightly larger 343 range of motion of the second phalangeal joint angle of the 3rd toe $(\beta)$ presented during slow 344 walking than running. In addition, the first phalangeal joint angle of the 4 th toe $(\varphi)$ flexed much 345 more at lift-off during running than slow walking. 

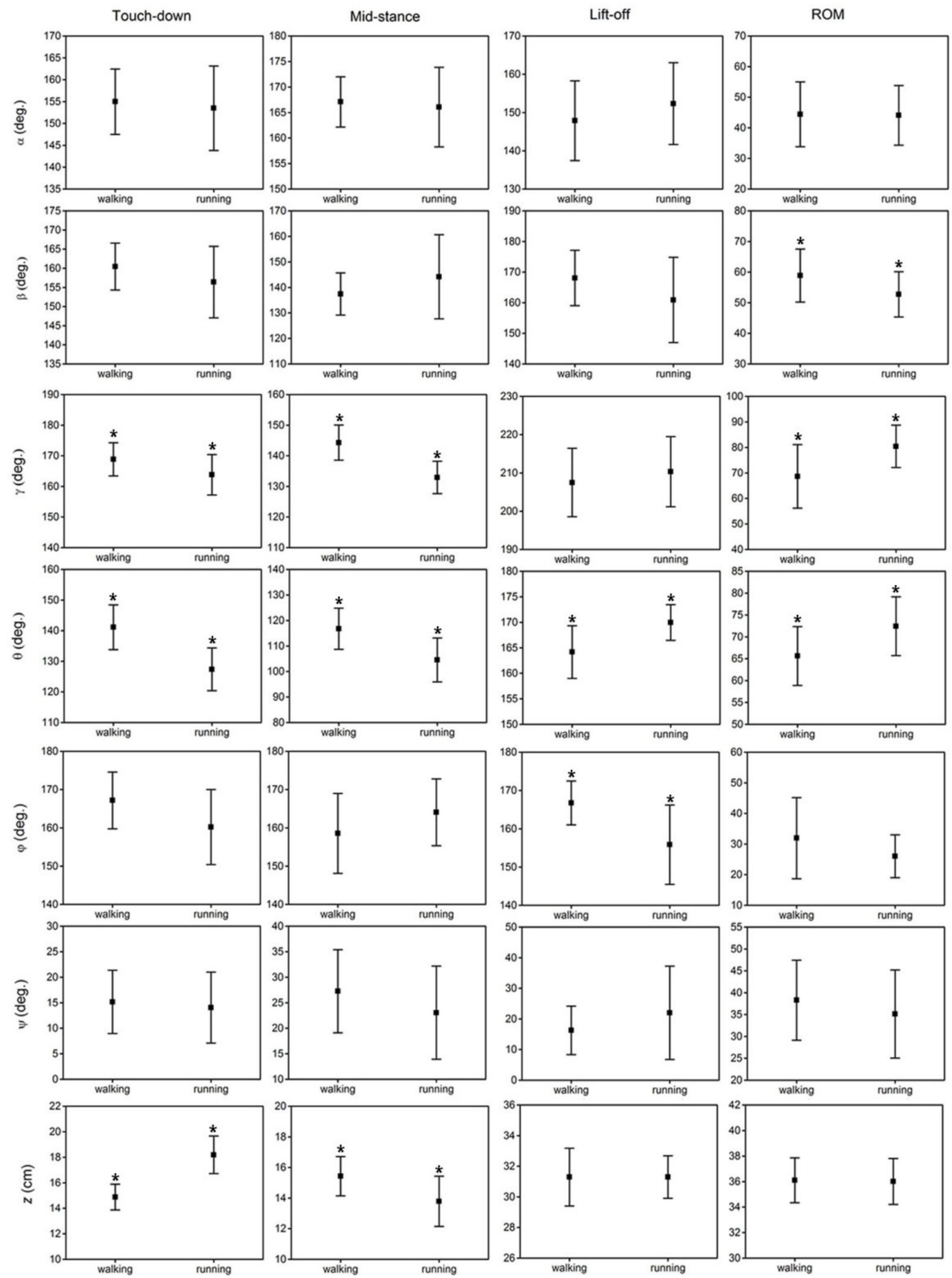

346 Figure 7. The averages and standard deviations of the six toe joint angles and the vertical displacement

347 of the metatarsophalangeal joint at touch-down, mid-stance, lift-off and also the ranges of motion during

348 slow walking and running. Statistically significant effect of locomotor gait are indicated by an asterisk (P $349<\mathbf{0 . 0 5})$. 
350 Figure 7 showed that statistically significant differences were found in several key indicators of 351 the MTP3 joint angle $(\gamma)$, the MTP4 joint angle $(\theta)$ and also the vertical displacement $(z)$ of the 352 metatarsophalangeal joint between slow walking and running trials. This was consistent with the 353 distinct patterns we observed in Figure 4. In addition, the MTP3 joint flexed much more at touch354 down, mid-stance, and used a larger range of motion during running compared to slow walking. 355 The MTP4 joint presented a more flexed positions at touch-down and mid-stance, and a more 356 extended position at lift-off during running. This led to a larger range of motion at the MTP4 joint 357 in running trials. Therefore, the MTP joint had a greater range of motion during running than that 358 in slow walking, which may further explain the metatarsophalangeal joint played an important 359 role as the energy storage and shock absorption during fast locomotion (Schaller et al., 2005; 360 Rubenson et al., 2007). For the vertical displacement of the metatarsophalangeal joint, though 361 very similar ranges of motion were used during slow walking and running, the 362 metatarsophalangeal joint was at a statistically higher position at mid-stance during slow walking, 363 which was consistent with the viewpoint that the metatarsophalangeal joint was positioned closer 364 to the ground, as speed increased (Schaller et al., 2009).

\section{Discussions}

366 This study first presents toe joint kinematic analysis in sub-adult ostriches during overground 367 slow walking and running. Reliable data on major toe joint angle trajectories and 368 metatarsophalangeal vertical displacement were obtained base on a large number of trials, 369 allowing interpretation of toe function in this flightless, cursorial bird with a unique elevated 370 metatarsophalangeal joint supporting only by two toes. Having chosen two genetically unrelated 371 subjects of the same sex and very similar age and size, the consistency of inter-individual results 372 in slow walking and running trials accurately document a generalized pattern in ostrich 373 locomotion. However, ostrich maturity has not been considered in our study. Some studies 374 suggested that scaling of kinematic variables largely agreed with predicted scaling for increasing 375 size. This demonstrated that there was close relationship for dynamic similarity between sub376 adult and adult ostriches (Smith et al., 2010). Ontogenetic scaling of locomotor mechanics largely 377 resulting from simple scaling of the limb segments rather than postural changes. 
378 Ostrich toes might play essential role in force and power generation, and also energy saving for

379 slow walking and running gaits (Schaller et al., 2009, 2011). Our study reveals that almost all the

380 six major toe joints present notably large motions from slow to fast locomotion. The MTP3 and

381 MTP4 joints exhibit the largest range of motion among all the six toe joints with an average range

382 of motion about 70 degree in slow walking and a higher motion range of 80 degree during

383 running. The smallest range of motion is found at the first phalangeal joint of the 4 th toe, but still

384 has an average range of about 30 degree. Rough skins, sturdy ligaments, fascia and lumpy fat

385 pads envelop the metatarsophalangeal joints, toe skeleton and interphalangeal joints to ensure

386 structural integrity, seemingly providing limitation on toe mobility (Schaller et al., 2011). In

387 addition, the lower hindlimb and multi-jointed muscle tendon system are primarily activated by

388 long tendons that store and release elastic energy during fast locomotion to provide an energetic

389 advantage (Schaller et al., 2011). The unique posture of the supra-jointed metatarsophalangeal

390 joint elastic energy storage structures is primarily maintained by ligaments (Schaller et al., 2009).

391 The metatarsophalangeal joint likely stores and releases elastic energy during fast locomotion

392 than slow walking gait.

\section{Two toes as an "integrated system"}

395 The 3rd toe and claw essentially forming an extension of the tarsometatarsal limb sustain most of

396 the impact force at touch-down and ensure stable load bearing and grip during stance phase

397 (Schaller et al., 2011). During slow walking and running, just after touch-down, simultaneous

398 flexions at the first phalangeal joint of the 3rd toe and the MTP3 joint, and also an extension at

399 the second phalangeal joint of the 3rd toe occurs implying compliance at the interphalangeal

400 joints of the 3rd toe is used to moderate ground impact at touch-down. Thereafter, the second

401 phalangeal joint remains fully extended in contact with the ground surface whereas the first

402 phalangeal joint flexes gradually till late stance. Interestingly, the third phalangeal joint angle of

403 the 3 rd toe $(\alpha)$ and the second phalangeal joint angle of the 3rd toe $(\beta)$ show statistically very

404 similar motion patterns during running compared to slow walking. Since no intrinsic muscles

405 exist in ostrich toes (Gangl et al,. 2004), this suggests that the tensions are well tuned by at the

406 toe flexors and extensors tendons crossing the different joints come from the same digital flexor

407 muscle during running, not only to counteract the higher ground reaction forces but also to

408 regulate the interphalangeal joint motions (Schaller et al., 2011). 
409 The first phalangeal joint of the 4th toe presents the largest motion variability among all the six 410 toe joints examined in this study with no obvious patterns found during slow walking and 411 running. This appears to support the previous hypothesis proposed by Schaller that the 4th toe 412 acts as a stabiliser to compensate uneven ground surface and adjust potential body imbalance 413 (Schaller et al., 2011). This seems further supported by the results that the deviation of the motion 414 range of the the first phalangeal joint angle of the 4th toe $(\varphi)$ during slow walking is almost 415 twice higher than that of running. Slow moving may need a greater level of neural control and 416 muscular regulation of the ground contact elements (Kummer, 1959; Schaller et al., 2011). The 417 angle between the first phalanges of the 3rd and 4th toes $(\psi)$ shows very similar pattern during 418 slow walking and running implying the high stiffness of the interphalangeal ligaments connecting 419 the $3 \mathrm{rd}$ and 4 th toes. Besides that, in order to measure and observe the 4th phalangeal joint 420 motion, we considered the phalanges IIIIIV and V of the 4th toe as one segment. However, there 421 may be some smaller motions within this simplified segment as well as a high sensitivity to marker placement. Therefore, this is probably one of the reasons that the first phalangeal joint of the 4th toe shows the largest motion variability. Over most of the duration when the 4th toe is in contact with the ground during slow walking, the average angle between the first phalanges of the 3rd and 4th toes $(\psi)$ is only about 25 degree. This is much lower than the maximum angle (34 degree) determined by a fresh anatomical dissection study (Schaller et al., 2011) and also the in vivo maximum value ( $46 \pm 8$ degree) recorded in this study. The average angle between the first phalanges of the 3 rd and 4 th toes $(\psi)$ further drops to about 20 degree during running. Schaller et al suggested that the 4th toe presumably allows compensation for uneven ground conditions to correct potential imbalances in CoM (center of mass), particularly at slower speeds (Schaller et al., 2011). In addition, the significantly lower variation in load distribution when running illustrates the effects of dynamic stability, which reduces the demand for fine adjustment at the 4th toe (Schaller et al., 2011). This strongly suggests that the body stabilization function of the 4th toe due to its lateral orientation may be as pronounced as proposed by the previous study especially for fast locomotion (Schaller et al., 2011). The major function of the 4th toe might be to offset the ground impact and reaction forces during early and middle stances, thereby to provide extra support for the lateral stability of the elevated metatarsophalangeal joint as the body weight transfers laterally. 
439 Although the interphalangeal joints of the 3rd and the 4th toes present distinct motion patterns in 440 stance phases of slow walking and running, the two major joints (MTP3 and MTP4 joint) 441 connecting the two toes to the tarsometatarsus share highly similar patterns for both slow and fast 442 locomotion (see Figure 4C and D). The average trajectories of the MTP3 joint angle $(\gamma)$ and the 443 MTP4 joint angle $(\theta)$ are almost perfectly in phase over the entire stance phases. This strongly 444 suggests that the 3rd and 4th toes actually move as an "integrated system" from slow to fast 445 locomotion. This synchronous pattern is more pronounced during running when the 4th toe lies 446 more closely to the 3rd toe due to a smaller average angle between the 3rd and 4th toes $(\psi)$ 447 during most of stance phase. Moreover, from our high speed videos of running trials, we found 448 that after the 4th toe clears off the ground, it aligns in a line and almost forms a single segment 449 with the 3 rd toe at push-off. This highly concerted toe motion is probably an emerging result of 450 the dynamic interaction of the proximal leg musculature, the distal passive ground contact 451 apparatus and the external environment. The leg muscles of ostriches are highly concentrated at 452 the proximal joints resulting in reducing moment of inertia with respect to the proximal joints. 453 This enables ostriches to achieve high step frequency energy efficiently (Schaller et al., 2011; 454 Haughton, 1865). The permanently elevated metatarsophalangeal joint further increases the leg 455 length thereby leading to higher stride length (Rubenson et al., 2007; Schaller et al., 2011). Even 456 though no intrinsic muscles are present to delicately regulate the toe motions (Gangl et al,. 2004), 457 our toe joint motion data strongly suggests that the toe joints are appropriately controlled by well458 tuned tensions at toe flexor and extensor tendons. Indeed, the ostrich intertarsal joint can be 459 considered as a passive mechanism well regulated by distal limb tendons and ligaments to work 460 as an "integrated system" to generate ground reaction forces, attenuate ground impacts and 461 accommodate ground surfaces whilst ensuring the stability of the elevated metatarsophalangeal 462 joint from slow to fast locomotion.

\section{Different strategies at slow and fast locomotion}

464 Our measurement data indicates that significantly different time history patterns are used by ostriches in the vertical displacement of the metatarsophalangeal joint and also the joint motions at MTP3 and MTP4 joints during running compared to slow walking. This is supported by the statically significant differences found in a number of key indicators of the displacement $z$, the MTP3 joint angle $(\gamma)$ and the MTP4 joint angle $(\theta)$. In most of stance phase of slow walking $469(10 \%-80 \%)$, the metatarsophalangeal joint only moves slightly upwards mainly due to the 
470 flexion of the first phalangeal joint of the 3rd toe because both the MTP3 and MTP4 joints and 471 also the second phalangeal joint of the 3rd toe remain almost stationary over this period.

472 Previous study revealed that ostriches used an inverted pendulum gait at slow locomotion 473 (Rubenson et al., 2004). The out-of-phase pattern in the fluctuations of the potential and kinetic 474 energies allows for a high percentage of mechanical energy recovery at slow speeds, which are 475 typical of walking gait in bipedal species (Cavagna et al. 1976, 1977; Heglund et al. 1982; Muir 476 et al. 1996). Whereas, at fast locomotion (including grounded running and aerial running), 477 ostriches tend to use a bouncing gait by using the legs as a springy mechanism to store and regain energy characterized by a marked reduction in the phase difference between the potential and kinetic energies (Haughton, 1865; Alexander et al., 1979; Muir et al., 1996; Rubenson et al., 2004; Daley et al., 2006; Jindrich et al., 2007; Rubenson et al., 2010; Schaller et al., 2009, 2011; Andrada et al., 2013; Birn-Jeffery et al., 2014; Hutchinson et al., 2015, 2016). The distinct toe joint motions at slow and fast locomotion observed in this study are probably the direct result of the selective use of those two distinct energy strategies at different speed ranges. At low speeds, the metatarsophalangeal joint only moves slightly over most of the stance phase $(10 \%-80 \%)$ by mainly using the first interphalangeal joint motion at the 3rd toe. However, at high speeds, the metatarsophalangeal joint presents a typical loading and rebounding pattern over the stance phase by mainly using the significant flexion and extension motions at the MTP3 and MTP4 joints possibly regulated by the stretched toe flexor tendons. This suggests that at fast locomotion the toes also work as a springy element in series with the proximal limb to attenuate ground impact, generate supporting forces and also may save metabolic energy cost.

In addition, in our study ostrich running speed was much lower than $50 \mathrm{~km} / \mathrm{h}(\sim 13 \mathrm{~m} / \mathrm{s})$, thus toejoint motion may be different from the result of average speed $2.77 \mathrm{~m} / \mathrm{s}$. This is mainly because locomotion was initiated either by display of food at the end of the corridor or by the lead scientist moving ahead to compel the ostrich to follow in our experiments. Tested running speed was lower than that ostrich ran for surviving in the field. In our study, the toe-joint motion only aimed at slow running gait instead of the fast running observed in the field. We think that it would be interesting to investigate the phalangeal joint kinematics at top running speeds. 
500 Though they were sub-adult, we found that the male ostriches were very dangerous in the process 501 of training. Thus, we decided to give up these male ostriches to do experiments. In addition, 502 female ostriches were nature timid and afraid of researchers. On the runway $1.5 \mathrm{~m}$ high wire 503 mesh fence, some female ostriches always crashed into the fence and hurt hindlimb in the process 504 of running training. Finally, we had to choose two tractable female ostriches as experimental 505 objects. We believe that results of phalangeal joint kinematics for only two individuals may still 506 be valuable for ostrich toe study in the future.

\section{Perspectives}

508 The gait measurements in this study were conducted on solid level ground surface. Future work involves the investigation of the toe-joint motions when moving on rough terrain at slow and fast speeds close to $50 \mathrm{~km} / \mathrm{h}$, and also during sideways maneuvers. This would enable us to inspect gait motions and foot bio-mechanics of ostriches when moving in an experimental setup closer to their natural habitat. In addition, how does ostrich foot generate sufficient braking and propulsive forces on granular media is of high interest to us. Moreover, the driving mechanism of the ostrich toe complex with a unique supra-jointed posture might inspire development of innovative bipedal robots capable of running fast and economically as ostriches.

\section{Conclusion}

All the six major toe joints investigated in this study show noticeable motions from slow to fast gaits. The MTP3 and MTP4 joints present the largest range of motion whereas the first phalangeal joint of the 4th toe exhibits the largest motion variability. The interphalangeal joints of the 3rd and 4th toes show very similar motion patterns during slow walking and running. However, the MTP3 and MTP4 joints motions and also the vertical displacement of the metatarsophalangeal joint present significantly different patterns during running and slow walking. Because of the biomechanical requirements, ostrich is likely to select the inverted pendulum gait at low speeds and also the bouncing gait at high speeds to improve movement performance and energy economy.

Indeed, the motions of the MTP3 and MTP4 joints are highly synchronized across the entire speed range examined in this study. This strongly indicates that the 3rd and 4th toes actually work 
529 as the complementary load sharing element mainly to ensure the lateral stability of the

530 permanently elevated metatarsophalangeal joint.

531 Supplemental Information

532 Supplemental information for this article can be found on line at 533 https://peerj.com/manuscripts/12297/files/.

\section{References}

Abourachid, A. and Renous, S. (2000). Bipedal locomotion in ratites (Paleognatiform): examples of cursorial birds. Ibis. 142, 538-549.

Alexander, R. M. (1984). Elastic energy stores in running vertebrates. Amer. Zool. 24, 85-94.

Alexander, R. M. (1985). The legs of ostriches (Struthio) and moas (Pachyornis). Acta. Biotheor. 34, 165-174.

Alexander, R. M., Maloiy, G. M. O., Njau, R. and Jayes, A. S. (1979). Mechanics of running of the ostrich (Struthio camelus). J. Zool. 187, 169-178.

Almeida Paz, I. C. L., Mendes, A. A., Balog, A., Almeida, I. C. L., Martins, M. R. F. B., Vulcano, L. C. and Komiyama, C. M. (2008). Quality parameters of the tibiae and femora of ostriches. Barz. J. Poultry Sci. 10, 163-167.

Andrada, E., Nyakatura, J. A., Bergmann, F., \& Blickhan, R. (2013). Adjustments of global and local hindlimb properties during terrestrial locomotion of the common quail (coturnix coturnix). Journal of Experimental Biology, 216(Pt 20), 3906-3916.

Birn-Jeffery, A. V., Hubicki, C. M., Blum, Y., Renjewski, D., Hurst, J. W., and Daley, M. A. (2014). Don't break a leg: running birds from quail to ostrich prioritise leg safety and economy on uneven terrain. J. Exp. Biol. 217, 3786-3796.

Castanet, J., Rogers, K. C., Cubo, J. and Jacques-Boisard, J. (2000). Periosteal bone growth rates in extant ratites (ostriches and emu). Implications for assessing growth in dinosaurs. $C$. R. Acad. Sci. Paris, Sciences de la Vie. 323, 543-550.

Cavagna, G. A., Thys, H. and Zamboni, A. (1976). The sources of external work in walking and running. J. Physiol. Lond. 262, 639-657.

Cavagna, G. A., Heglund, N. C. And Taylor, C. R. (1977). Mechanical work in terrestrial locomotion: two basic mechanisms for minimizing energy expenditure. Am. J. Physiol. 233, R243-R261. 
559

560

561

562

563

564

565

566

567

568

569

570

571

572

573

574

575

576

Deeming, D. C. (2003). The Ostrich - Biology, Production and Health. Cambridge, UK: Cambridge University Press.

Daley, M. A., \& Aa., B. (2006). Running over rough terrain reveals limb control for intrinsic stability. Proceedings of the National Academy of Sciences, 103(42), 15681-6.

Fowler, M. E. (1991). Comparative clinical anatomy of ratites. J. Zoo. Wildlife Med. 22, 204227.

Gatesy, S. M. (1991). Hind limb scaling in birds and other theropods: implications for terrestrial locomotion. J. Morphol. 209, 83-96.

Gatesy, S. M. and Biewener, A. A. (1991). Bipedal locomotion: effects of speed, size and limb posture in birds and humans. J. Zool. 224, 127-147.

Gangl, D., Weissengruber, G. E., Egerbacher, M. and Forstenpointner, G. (2004). Anatomical description of the muscles of the pelvic limb in the ostrich (struthio camelus). Anat. Histol. Embryol. 33, 100-114.

Haughton, S. (1865). XXIX.- On the muscular mechanism of the leg of the Ostrich. J. Nat. Hist. 15, 262-272.

Heglund, N. C., Cavagna, G. A. and Taylor, C. R. (1982). Energetics and mechanics of terrestrial locomotion. III. Energy changes of the centre of mass as a function of speed and body size in birds and mammals. J. Exp. Biol. 79, 41-56.

Hutchinson, J. R., Rankin, J. W., Rubenson, J., Rosenbluth, K. H., Siston, R. A. and Delp, S. L. (2015). Musculoskeletal modelling of an ostrich (struthio camelus) pelvic limb: influence of limb orientation on muscular capacity during locomotion. Peerj. 3:e1001.

Jindrich, D. L., Smith, N. C., Jespers, K. and Wilson, A. M. (2007). Mechanics of cutting maneuvers by ostriches (Struthio camelus). J. Exp. Biol. 210, 1378-1390.

Kummer, B. (1959). Bauprinzipien des Säugerskeletts. Stuttgart: Georg-Thieme Verlag.

Muir, G. D., Gosline, J. M. and Steeves, J. D. (1996). Ontogeny of bipedal locomotion: walking and running in the chick. J. Physiol. 493, 589-601.

Rubenson, J., Heliams, D. B., Lloyd, D. G. and Fournier, P. A. (2004). Gait selection in the ostrich: mechanical and metabolic characteristics of walking and running with and without an aerial phase. Proc.R. Soc. Lond. B 271, 1091-1099.

Rubenson, J., Lloyd, D. G., Besier, T. F., Heliams, D. B. and Fournier, P. A. (2007). Running in ostriches (Struthio camelus): three-dimensional joint axes alignment and joint kinematics. J. Exp. Biol. 210, 2548-2562. 
591

592

593

594

595

596

597

598

599

600

601

602

603

604

605

606

607

608

609

610

Rubenson, J., Lloyd, D. G., Heliams, D. B., Besier, T. F. and Fournier, P. A. (2010). Adaptations for economical bipedal running: the effect of limb structure on threedimensional joint mechanics. J. R. Soc. Interface. 8, 740-755.

Rankin, J. W., Jonas, R., \& Hutchinson, J. R. (2016). Inferring muscle functional roles of the ostrich pelvic limb during walking and running using computer optimization:. Journal of the Royal Society Interface, 13(118).

Stoessel, A. and Fischer, M. S. (2012). Comparative intralimb coordination in avian bipedal locomotion. J. Exp. Biol. 215, 4055-4069.

Schache, A. G., Blanch, P. D., Dorn, T. W., Brown, N. A., Rosemond, D. and Pandy, M. G. (2011). Effect of running speed on lower limb joint kinetics. Med. Sci. Sports Exerc. 43, 1260-1271.

Schaller, N. U., D'Août, K., Villa, R., Herkner, B. and Aerts, P. (2011). Toe function and dynamic pressure distribution in ostrich locomotion. J. Exp. Biol. 214, 1123-1130.

Schaller, N. U., Herkner, B., Villa, R. and Aerts, P. (2009). The intertarsal joint of the ostrich (Struthio camelus): anatomical examination and function of passive structures in locomotion. J. Anat. 214, 830-847.

Schaller, N., D'Août, K., Herkner, B. and Aerts, P. (2007). Phalangeal load and pressure distribution in walking and running ostriches (Struthio camelus). Comp. Biochem. Physiol. A 146, S122.

Smith, N. C. and Wilson, A. M. (2013). Mechanical and energetic scaling relationships of running gait through ontogeny in the ostrich (Struthio camelus). J. Exp. Biol. 216, 841-849.

Smith, N. C., Jespers, K. J. and Wilson, A. M. (2010). Ontogenetic scaling of locomotor kinetics and kinematics of the ostrich (Struthio camelus). J. Exp. Biol. 213, 1347-1355.

Smith, N. C., Payne, R. C., Jespers, K. J. and Wilson, A. M. (2007). Muscle moment arms of pelvic limb muscles of the ostrich (Struthio camelus). J. Anat. 211, 313-324.

Smith, N. C., Wilson, A. M., Jespers, K. J. and Payne, R. C. (2006). Muscle architecture and functional anatomy of the pelvic limb of the ostrich (Struthio camelus). J. Anat. 209, 765779.

Watson, R. R., Rubenson, J., Coder, L., Hoyt, D. F., Propert, M. W. and Marsh, R. L. (2011). Gait-specific energetics contributes to economical walking and running in emus and ostriches. Proc. R. Soc. Lond. B 278, 2040-2046. 